\title{
Analysis of Actual Psychological Condition in Patients with Functional Intestinal Disorders
}

\begin{abstract}
N.P. Chernus ${ }^{1}$, Gorenkov R. B ${ }^{1}$, S.K. Sivkova ${ }^{1}$, A.S. Sivkov ${ }^{1}$, A.M. Zolotovickaja ${ }^{1}$, T. S. Savina ${ }^{1}$.
1 - I.M. Sechenov First Moscow State Medical University: Moscow, Russia

Background: Phenomenon of psychological mechanisms specific gravity/significance determination in the pathologic behavior of functional intestinal disorders of various forms has claimed attention from physicians and psychologists for the more than 40 years.

Methods: The study enrolled 114 patients (26 male and 88 female) with irritable bowel syndrome (IBS-D, IBS-C, IBS-M and IBS-U), functional constipation (FC) and functional diarrhea (FD). The average subject age was 43,6 11 years. In order to achieve the study objective, MMPI method was used, where the range between 30 and $70 \mathrm{~T}$-scores indicated normal psychological condition, while increased score by one or several clinical scales were deemed to signal the pathological profile.

Results: Analysis of averaged subject profiles showed that in most subjects the average profile score exceeded 80 T-scores, and namely in 64\% FC patients, 60\% IBS-M patients and 56\% IBS-C patients vs. 44\% IBS-U patients, $43 \%$ IBS-D patients and 33\% FD patients, $\mathrm{p}<0,01$. The average profile above $70 \mathrm{~T}$-scores, but below $80 \mathrm{~T}$-scores by one or several clinical scales was observed in $50 \%$ for FD vs. $32 \%$ for IBS-D; $p<0,01$; and twice as often for IBS-C: $33 \%$ vs. $16 \%$ for FC. In the lower part of continuum, where the psychological factor influence is relatively low, the profile distribution was as follows: $25 \%$ for IBS-D, 20\% for FC and IBS-M, $17 \%$ for FD and IBS-M, $11 \%$ for IBS-C.
\end{abstract}

Conclusion: The data obtained demonstrate the significant specific gravity of psychosomatic component in disease development, progression and chronization in patients with FC, IBS-M. 